\title{
Mobile Oriented Future Internet (MOFI): Architectural Designs and Experimentations
}

\author{
Seok-Joo Koh $\mathbb{D}$ \\ School of Computer Science and Engineering, Kyungpook National University, Daegu 41566, Korea; \\ sjkoh@knu.ac.kr; Tel.: +82-53-950-7356
}

Received: 22 April 2020; Accepted: 22 April 2020; Published: 23 April 2020

\section{Introduction}

With the explosive growth of smart phones and Internet-of-Things (IoT) services, the effective support of seamless mobility for a variety of mobile devices and users is becoming one of the key challenging issues. It is expected that the future Internet will be evolved toward 'mobile-oriented' [1]. In the mobile-oriented future internet (MOFI) environment, there will be a large number of mobile devices and users with a variety of heterogeneous mobile, wireless, sensor and vehicular networks.

To provide mobility management, a lot of protocols have so far been proposed, which include host identity protocol (HIP) [2], identifier-locator network protocol (ILNP) [3] and quick UDP internet connection (QUIC) [4]. However, these works may not be enough to provide mobility management in the MOFI environment. We may consider many recently proposed technologies, such as software defined networking (SDN) [5]. It is noted that SDN can be effectively used to control the network traffics by separating the control function from the packet data delivery function in the network. We also need to consider a variety of mobile networks in the MOFI environment, which include flying ad-hoc networks (FANET) [6] and connected vehicular networks [7].

\section{The Present Issue}

This special issue consists of seven papers that discuss how to enhance mobility management and its associated performance in the MOFI environment. The first two papers deal with the architectural design and experimentation of mobility management schemes, in which new schemes are proposed and the real-world testbed experimentations are performed. The subsequent three papers focus on the use of SDN for effective service provisioning in the MOFI environment, together with the real-world practices and testbed experimentations. The remaining two papers discuss the network engineering issues in the newly emerging mobile networks, such as FANET and connected vehicular networks.

In Reference [8], to overcome the drawbacks of the existing centralized mobility management schemes, the MOFI architecture is designed, which includes the separation of data and control planes for getting an optimal data path, and the distributed identifier-locator mapping control for alleviating traffic overhead at a central agent. In this work, the validity of the MOFI architecture is evaluated through the experimentations over the European Union (EU)-Korea testbed network. Reference [9] proposes an enhanced mobility management scheme in the ILNP-based mobile networks, in which the identifier-locators (ID-LOCs) mappings are managed in the fully distributed manner by using a mobile dynamic domain name system (m-DDNS) server located in each domain.

The SDN technology can be used for performance enhancement in the MOFI environment. Reference [10] presents an SDN-based quality of experience (QoE) control scheme for dynamic adaptive streaming over HTTP/3 (DASH), using the quick UDP internet connection (QUIC) [4] for mobile devices and users. Reference [11] discusses the testbed experimentations of SDN using SmartX boxes that are distributed across multiple sites. Each SmartX box consists of several virtualized functions that are 
categorized into SDN and cloud functions. Multiple SmartX boxes are deployed and inter-connected through SDN in the distributed environments. Reference [12] discusses the resource management scheme in the mobile cloud environment, which exploits SDN to introduce a vendor-agnostic concept.

The MOFI environment may consist of various types of emerging mobile networks, such as FANET and connected vehicular networks. FANET is an ad-hoc network for data transfer among the unmanned aircrafts in the three-dimensional space. Reference [13] proposes a routing scheme for FANET which can adapt to rapid changes in network topology and effectively improve the network performance. In Reference [14], the connected vehicular networks are considered, in which a new intersection signal control model is proposed based on vehicle-to-infrastructure (V2I) communication, and the simulation analysis is made with the real-world data.

\section{Future}

A variety of research works have so far been made with some testbed experimentations in the MOFI environment, as addressed in this special issue. However, some challenges still remain for further study in the future. New architectural models for MOFI need to be investigated by considering a variety of mobile devices/users for IoT services. The existing mobility management protocols, such as HIP and ILNP, can be reviewed and compared to design a more effective mobility management scheme. Some more testbed experimentations are also required for validation of the schemes for MOFI in real-world networks. The relevant standardization activities need to be investigated and initiated, if necessary, in the associated standards-defining organizations, such as ITU-T, ISO, JTC1, IETF, etc.

Author Contributions: S.-J.K. managed the whole editorial process of the special issue, 'Mobile Oriented Future Internet (MOFI): Architectural Designs and Experimentations', published by journal Electronics. He also drafted this editorial summary. All authors have read and agreed to the published version of the manuscript.

Acknowledgments: First of all, I would like to thank all researchers who submitted articles to this special issue for their excellent contributions. We are also grateful to all reviewers who helped in the evaluation of the manuscripts and made very valuable suggestions to improve the quality of contributions. We would like to acknowledge the editorial board of Electronics, who invited me as a guest editor to this special issue. We are also grateful to the Electronics Editorial Office staff who worked thoroughly to maintain the rigorous peer-review schedule and timely publication.

Conflicts of Interest: The author declares no conflicts of interest.

\section{References}

1. Kim, J.-I.; Jung, H.; Koh, S.-J. Mobile Oriented Future Internet (MOFI): Architectural Design and Implementations. Etri. J. 2013, 35, 666-676. [CrossRef]

2. Moskowitz, R.; Nikander, P.; Jokela, P.; Henderson, T. Host Identity Protocol. IETF Request Comments (RFC) 2008, 5201, 1-102.

3. Atkinson, R.J.; Bhatti, S.N. Identifier-Locator Network Protocol (ILNP) Architectural Description. IETF Request Comments (RFC) 2012, 6740, 1-53.

4. Iyengar, J.; Thomson, M. QUIC: A UDP-Based Multiplexed and Secure Transport, IETF Internet Draft, Draft-IETF-Quic-Transport-27. 2020. Available online: https://www.ietf.org/id/draft-ietf-quic-transport-27. txt (accessed on 30 March 2020).

5. Xia, W.; Wen, Y.; Foh, C.H.; Niyato, D.; Xie, H. A Survey on Software-Defined Networking. IEEE Commun. Surv. Tutor. 2015, 17, 27-51. [CrossRef]

6. Bekmezci, I.; Sahingoz, O.K.; Temel, S. Flying Ad-Hoc Networks (FANETs): A survey. Ad Hoc Netw. 2013, 11, 1254-1270. [CrossRef]

7. Lu, N.; Cheng, N.; Zhang, N.; Shen, X.; Mark, J.W. Connected Vehicles: Solutions and Challenges. IEEE Internet Things J. 2014, 1, 289-299. [CrossRef]

8. Kim, J.-I.; Choi, N.-J.; You, T.-W.; Jung, H.; Kwon, Y.-W.; Koh, S.-J. Mobile-Oriented Future Internet: Implementation and Experimentations over EU-Korea Testbed. Electronics 2019, 8, 338. [CrossRef]

9. Gohar, M.; Choi, J.-G.; Ahmed, W.; Rahman, A.U.; Muzammal, M.; Koh, S.-J. Distributed Identifier-Locator Mapping Management in Mobile ILNP Networks. Electronics 2020, 9, 58. [CrossRef] 
10. Guillen, L.; Izumi, S.; Abe, T.; Suganuma, T. SAND/3: SDN-Assisted Novel QoE Control Method for Dynamic Adaptive Streaming over HTTP/3. Electronics 2019, 8, 864. [CrossRef]

11. Risdianto, A.C.; Usman, M.; Kim, J.-W. SmartX Box: Virtualized Hyper-Converged Resources for Building an Affordable Playground. Electronics 2019, 8, 1242. [CrossRef]

12. Abbasi, A.A.; Al-qaness, M.A.A.; Elaziz, M.A.; Hawbani, A.; Ewees, A.A.; Javed, S.; Kim, S. Phantom: Towards Vendor-Agnostic Resource Consolidation in Cloud Environments. Electronics 2019, 8, 1183. [CrossRef]

13. Hong, J.; Zhang, D. TARCS: A Topology Change Aware-Based Routing Protocol Choosing Scheme of FANETs. Electronics 2019, 8, 274. [CrossRef]

14. Ren, C.; Wang, J.; Qin, L.; Li, S.; Cheng, Y. A Novel Left-Turn Signal Control Method for Improving Intersection Capacity in a Connected Vehicle Environment. Electronics 2019, 8, 1058. [CrossRef]

(C) 2020 by the author. Licensee MDPI, Basel, Switzerland. This article is an open access article distributed under the terms and conditions of the Creative Commons Attribution (CC BY) license (http://creativecommons.org/licenses/by/4.0/). 